\title{
La honestidad, la amistad y la autoestima: valores que promueven el clima relacional positivo
}

DOI: $10.46932 /$ sfjdv2n1-011

Received in: November 1st, 2020

Accepted in: December 30th, 2020

\author{
Abraham Bernárdez-Gómez \\ Contratado Predoctoral FPI. Ref: BES-2017-081040 \\ Universidad de Murcia. \\ E-mail: abraham.bernandez@um.es \\ José Santiago Álvarez-Muñoz \\ Dr. en Educación \\ Universidad de Murcia \\ E-mail: josesantiago.alvarez@um.es \\ María Luisa Belmonte \\ Dra. en Investigación e Innovación Educativa \\ Universidad de Murcia \\ E-mail: marialuisa.belmonte@um.es
}

\section{RESUMEN}

Las relaciones en el entrono escolar y en la comunidad educativa han sido y se presentan en la actualidad como una de las principales preocupaciones para los gestores y lideres educativos. Ello se debe a la profunda relación que guarda con el bienestar y el rendimiento académico como se ha podido comprobar a lo largo de la investigación anterior estudiada. Por ese motivo se ha planteado la necesidad de establecer un plan de intervención en el que se propondrá una serie de actividades que trabajen diferentes tipos de valores, dando cuenta de la importancia de cada uno de ellos y su beneficio al desarrollo socioeducativo y personal de los estudiantes y a la implicación en la comunidad educativa mediante un clima relacional positivo. Esta acción se lleva a cabo dentro de una propuesta de intervención educativa en el programa de Cruz Roja: "Éxito escolar", en la Comunidad Aútonoma de la Región de Murcia, España.

Palabras claves: Valores, honestidad, amistad, autoestima.

\section{INTRODUCCIÓN}

Tiempo atrás, el contexto y la institución escolar se ha venido centrando principalmente en la transmisión de conceptos y contenidos estructurados. De manera paulatina esto ha ido modificándose con el paso del tiempo, ya que se ha contemplado la necesidad de perpetuar y difundir una serie de valores personales y sociales que permitan a los más jóvenes desenvolverse en una sociedad globalizada y en constante cambio. 
Actualmente, la educación en valores es uno de los problemas estratégicos más representativos dentro de la enseñanza-aprendizaje, suscitando gran interés social y didáctico, formando parte incluso del contenido específico en los currículos escolares de todos los niveles educativos (Parra, 2003).

La metamorfosis que ha sufrido la relación familia-escuela está justificada por la acelerada transformación a la que la sociedad se ha visto sometida, y por los continuos cambios que han influido en ella con el paso de los años. Así pues, la institución escolar como claro agente socializador, debe ser consciente de las necesidades emergentes que derivan de toda esta evolución, atendiendo así los nuevos requisitos que a las familias se le plantean (Martínez, 2014), las cuales "no tienen nada que ver con las generaciones que nos preceden" (González, 2014, p. 28).

Estos factores, junto con la educación intercultural y la integración de los chicos de diferentes orígenes que llegan a España, es uno de los mayores desafíos que debe afrontar nuestro sistema educativo (Santos y Lorenzo, 2009).

\subsection{LA EDUCACIÓN EN VALORES}

"Los centros educativos han experimentado grandes cambios en las últimas décadas, tendientes hacia la heterogeneidad, multiculturalidad, diversidad de capacidades, de contextos familiares, así como otros aspectos asociados con la ciudadanía y las experiencias vitales, que marcan cognitiva, conductual y emocionalmente a los educandos" (Hernández-Prados et al., 2017, p.90). Hoy en día, la educación representa una acción esencial en el desarrollo de la ciudadanía y en el progreso hacia un entorno democrático (Martínez, 2006). De manera global, socialmente hablando, es comúnmente aceptada la idea de que la institución escolar, tal y como es concebida hoy día, necesita una remodelación para favorecer la mejora de los procesos de enseñanza-aprendizaje del alumnado (Booth y Ainscow, 2000). Con el desarrollo económico y tecnológico actual, el hombre ha tenido que enfrentar, según algunos especialistas, una crisis de valores (Rivas, 1996). "En la actualidad, la importancia del problema de los valores es innegable; la crisis del hombre actual es una crisis de valores que requiere un examen crítico, a fondo, de su naturaleza, sentido, fundamento y jerarquía" (González, 1991, p.145).

Los niños actualmente se están viendo envueltos en una clara pérdida de valores, preocupando esta situación tanto a padres como a profesores que se ven desprovistos de las herramientas necesarias para solventar este problema (Mínguez, 2014). Esta pérdida de valores en la sociedad actual se debe a una gran variedad de factores como la falta de una educación adecuada donde se dé una buena inculcación de buenas prácticas y actitudes, tanto dentro del contexto escolar, como en el seno familiar y los medios de comunicación, entre otros. En este sentido, los niños reciben constantemente mensajes contradictorios de la escuela, familia, los amigos y los medios de comunicación. Mientras la escuela transmite unos 
determinados valores (Trillo, Parada y Bernárdez-Gómez, 2020), los medios de comunicación transmiten otros que son totalmente contradictorios (Bolívar, 1995, 2006). El hecho de incluir los valores en educación plantea a su vez nuevas exigencias, ya que es necesario incorporar otros contenidos y unas nuevas competencias. Además de esto, según Ortega et al., (1996) este tipo de educación en valores implica exigencias como someter a evaluación lo que ya estaba presente en las aulas a un nivel no-formal, mediante actividades capaces de producir este cambio.

Es preciso señalar que esta pérdida de valores en España está vigente desde hace décadas, sobre todo, se pudo observar más notoriamente con la ampliación de los derechos y libertades individuales del Estado Liberal, que supuso la pérdida del sentido de la obediencia a toda norma y la fomentación de una mentalidad individualista y preocupada por una satisfacción hedonista (Parra, 2003). Con el período de la transición democrática, se introdujo por primera vez, en la educación general básica (EGB) la asignatura de Educación para la convivencia, con el objetivo de empezar a trabajar con los alumnos una serie de valores en el aula. No obstante, no se produjo en España la idea de una buena educación en valores hasta la aparición de la Ley orgánica general del sistema educativo (LOGSE, 1990) donde en su preámbulo y respectivos artículos se recoge la idea de la transmisión de valores que hagan posible la vida en la sociedad y se configuren los valores como la tolerancia y la libertad recogidos en la Constitución de 1978.

Con las dos últimas leyes educativas, la ley orgánica de educación (LOE, 2006) y la actual ley orgánica para la mejora de la calidad educativa (LOMCE, 2013) se recogen en sus artículos destinados a la etapa de Educación Primaria la preocupación por la formación para la paz, el respeto a los derechos humanos, la vida en común, la cohesión social, el conocer y apreciar los valores y las normas de convivencia, entre otros valores.

A pesar de que se recojan en la actual legislación educativa el trabajar con los valores, no se observa hoy en día que, en la puesta en práctica en el aula, se esté llevando a cabo de forma satisfactoria. Cada sociedad, en un momento determinado de su historia, establece los valores que considera necesarios y adecuados para dar cabida a las necesidades sociales que se plantean es ese preciso momento. La escuela es, por excelencia, la institución encargada de la transmisión e impulso de dichos valores, por medio de la actividad educativa, desarrollada en sus aulas y contexto en general. Esta educación que se ofrece es, entre otras cosas, habilidades y actitudes que son considerados relevantes y que demanda un grupo social. Así, pues, todo proceso educativo está relacionado con la transmisión de valores (Parra, 2003). Es a través de la educación, cómo la sociedad se ha venido perpetuando, por medio de la influencia de éstos en un grupo de iguales, que ha configurado así un determinado estándar de vida (Parra, 2003).

Carreras en 1995 defendía que una enseñanza sin principios educativos y sin valores sería un completo error, ya que no seríamos capaces de encontrar el sentido de la vida. Atendiendo a la definición 
acerca del concepto de valor que otorgan Ballester y Calvo (2007), vemos como estos son los principios a seguir en las situaciones aceptadas como deseables para satisfacer las necesidades humanas, ya sean en grupo o de forma colectiva, debiendo estar reflejadas en las pautas de comportamiento del alumno en este caso.

Desde un punto de vista filosófico, los valores han sido estudiados a través de la filosofía práctica. Autores como Platón, Sócrates o Aristóteles, concebían la sabiduría, la justicia, el amor, o la verdad, entre otros, como algunos de los valores imprescindibles paras vivir plena y honestamente, en armonía social (Hessen y Romero, 1970; Deleuze, 1984). Un aporte interesante sería el que Fabelo (2003) defiende, acerca de cómo los valores podrían ser considerados como las características que suplen en el contexto social la función que en otras especies desempeñan los instintos biológicos. Así pues, el simple hecho de que el género humano haya sido capaz de poner en peligro su propia supervivencia, es un indicador claro de la necesidad del mismo de tomar conciencia de las necesidades y transformaciones que precisa. Por otro lado, para la sociología, el concepto de valor es referido al significado comunitario que tiene la realidad y todo lo que la compone, para una determinada estructura, grupo social o individuo, en la medida en que entran en relación con las necesidades de los mismos (Porto et al., 2009).

Educar en valores es la respuesta a una educación democrática y participativa. Según postulaba Camps (1998), es de vital importancia promover una educación en valores relacionados con los Derechos Humanos, como la libertad, igualdad, justicia, solidaridad, tolerancia, responsabilidad, participación, etc., para que estas actitudes generen comportamientos interiorizados que ayuden a la resolución de los posibles problemas dentro del contexto escolar o social. Otros ejemplos de valores necesarios en la sociedad actual son los propuestos por Tierno (2000), como la autenticidad, la dignidad, la sinceridad y la coherencia, la justicia, la honradez, la fidelidad y el civismo o buenas maneras y la familia, etc. Este autor asegura que estos valores atraviesan momentos difíciles y es preciso volver a otorgarles protagonismo, ya que son necesarios para la propia autorrealización del individuo y para su contexto.

Según el contexto en el que nos veamos inmersos, prevalecen más una serie de valores que otros, aunque los más recomendables para aplicar en la práctica serían aquellos que contribuyen no sólo al bien individual sino también al bien común. Sólo de esta manera, se podrá convivir en una sociedad verdaderamente democrática (Beane y Apple, 2002), educando por y para la ciudadanía de los educandos desde un punto de vista no sólo académico, sino sobre todo desde la práctica educativa (Bernárdez-Gómez y Belmonte, 2020a, 2020b; Naval y Laspalas, 2000; Bartolomé et al., 2002; Annaut, 2002; Rodríguez, 2002). Interiorizar de manera significativa valores basados en principios democráticos o cívicos, nos acerca a esa condición deseada de ciudadanos plenos dentro de una sociedad (Camps y Giner, 2004). 
Aquellos valores que desarrollen la personalidad del alumno para su mayor integración en el contexto escolar, o social, potenciarán también de esta manera su felicidad.

Es preciso señalar que los valores que realmente más influyen en la vida (Bernárdez-Gómez, 2021) son los que cada persona consigue construir por sí misma, a través de interacciones críticas con el mundo y la cultura (Parra, 2003). Autores como Ortega et al., (1996) postulan que, desde los valores, el niño tiene la oportunidad de pensar y actuar, siendo él mismo el que toma las decisiones, dando explicación y coherencia a su vida. Además, estos valores no suceden paralelamente a ellos, sino que son interiorizados, necesitando de la experiencia para poder enseñarlos-aprenderlos. Sólo de esta manera podrán apropiarse de ellos.

Por todo, en todo tiempo y lugar, la escuela ha contribuido, de forma decisiva, al proceso de socialización de las generaciones más jóvenes en los valores comunes, compartidos por el grupo social, con el fin de garantizar el orden en la vida social y su continuidad (Hernández y Álvarez, 2019). Si la transmisión de unos valores considerados como fundamentales, era indispensable ya en las sociedades tradicionales con el fin de preservar sus tradiciones y sus formas de vida, cuanto más complejas y plurales son las sociedades, como acontece en las sociedades democráticas actuales, tanto más necesaria se hace la tarea de una educación en valores para el mantenimiento de la cohesión social (Parra, 2003).

En muchos centros escolares de España se están llevando a cabo programas y proyectos relacionados con la temática de educación en valores. En concreto, en la Región de Murcia encontramos programas de educación en valores personales y sociales para los niños de educación infantil, mediante metodologías innovadoras, entre los cuales se destacan, el orden, la responsabilidad, la paz, la igualdad, la amistad, la autoestima y el esfuerzo. Por otro lado, Esteban (2003) nos dice que en los centros educativos se deben promover tanto los valores dentro del aula como fuera de esta, con el objetivo de formar personas autónomas. Trabajando en los alumnos la capacidad de diálogo para resolver situaciones conflictivas, enseñándoles para ello a pensar de forma crítica y respetando los Derechos Humanos. Estos programas buscan satisfacer la necesidad de paliar la crisis de valores en los centros escolares desde edades tempranas.

\section{PLANIFICACIÓN METODOLÓGICA SOBRE LOS VALORES}

\subsection{LA AUTOESTIMA}

\subsubsection{Objetivos propuestos a tratar en las actividades:}

- Fomentar cualidades de atención, escucha y diálogo como medios y como clima fundamental para lograr unas relaciones interpersonales óptimas y una solución no violenta de los posibles conflictos que pueden surgir. 
- Mejorar el clima de trabajo, fomentando la actitud participativa y solidaria de la comunidad educativa.

\subsubsection{Actividad 1 sobre la autoestima:}

Para trabajar con los alumnos el valor de la autoestima, se les repartirá a todos los alumnos un folio en blanco para que dibujen un árbol, con sus raíces, ramas, hojas y frutos y se les explicará que en las raíces escribirán las cualidades positivas que cada uno cree tener, en las ramas, las cosas positivas que hacen y en los frutos, los éxitos o triunfos que han logrado hasta ahora. Para que se trabaje de forma efectiva con los alumnos que pertenecen al primer curso de Primaria, es imprescindible la colaboración en esta actividad de las profesoras voluntarias de apoyo escolar para que les ayude a identificar cuáles son sus cualidades positivas, y estar atentas a las posibles necesidades que les puedan surgir durante el desarrollo de esta actividad. Una vez terminen, deben escribir su nombre y sus apellidos en la parte superior del dibujo. Seguidamente, los alumnos se colocarán el árbol en el pecho, de forma que el resto de sus compañeros puedan verlo y uno por uno se situará en el centro de la clase para que les diga a todos los presentes en el aula cuáles son las cualidades positivas que cree que posee, las cosas que se les da bien, así como sus triunfos tanto académicamente como fuera del contexto escolar.

\subsubsection{Actividad 2 sobre la autoestima:}

El aula se dividirá en grupos formando un círculo. A cada uno de los integrantes de los grupos se les dará un papel para que anoten adjetivos, de los cuales, la mitad deben caracterizarles a ellos mismos negativamente y la otra mitad corresponder a un adjetivo positivo por cada integrante de su círculo. Cuando hayan terminado de escribir cada uno de ellos los adjetivos, leerán los integrantes de cada círculo los adjetivos que se han atribuido a ellos mismos y los compararán con lo que han asignado sus compañeros. Todo esto, con el fin de que ellos mismos se den cuenta de que a veces lo que ellos piensan de sí mismos no se corresponde con lo que piensan los demás.

\subsection{LA AMISTAD}

\subsubsection{Objetivos propuestos a tratar en las actividades:}

- Fomentar cualidades de atención, escucha y diálogo como medios y como clima fundamental para lograr unas relaciones interpersonales óptimas y una solución no violenta de los posibles conflictos que pueden surgir.

- Mejorar el clima de trabajo en el aula donde se lleva a cabo el apoyo escolar. 


\subsubsection{Actividad 1 sobre la amistad:}

Se comenzará con el visionado de una escena de un capítulo de dibujos animados titulado: el valor de la amistad, donde aparece claramente reflejado cómo las personas van construyendo "muros" entre ellas que imposibilita poder relacionarse con normalidad, quizás solamente por tener puntos de vista distintos. Se le realizará al alumnado las siguientes preguntas:

1. ¿Qué habéis aprendido de este cortometraje?

2. ¿Creéis que es bueno construir "muros" entre las personas?

3. ¿Se debe rechazar a un amigo porque piensa distinto a nosotros?

4. ¿Qué valores creéis que aparecen en esta pequeña escena?

Entre todos, levantando la mano, contestarán las preguntas y a continuación, se irán todos ellos al aula donde formarán grupos y se les dará hojas blancas donde se dibujará en el centro un corazón rojo. Cada miembro del grupo deberá pensar en sus amigos y escribir dentro del corazón las cosas buenas que les aporta esa amistad y qué es aquello que no les gusta de sus amigos. Posteriormente, todos juntos pondrán en común aquellos aspectos positivos que le aportan la amistad y, por otro lado, las cosas negativas. Una vez puesto estas ideas en común, se les explicará antes de finalizar esta actividad a todos los alumnos que, a pesar de no gustarnos ciertas cosas de nuestros amigos, siempre hay que valorar la amistad y centrarnos más en las cosas positivas que nos aporta la relación con los demás, en lugar de lo negativo, siempre bajo la dinámica del respeto.

\subsubsection{Actividad 2 sobre la amistad:}

Para finalizar leerá el grupo de los más pequeños en voz alta el cuento de Los músicos de Bremen, para trabajar su competencia lectora y para que tomen protagonismo en el aula. Tras su lectura, se realizará lo siguiente:

En primer lugar, todos los alumnos harán un resumen del cuento y le preguntaremos:

1. ¿Qué animales aparecen en el cuento?

2. ¿Son amigos o sólo son compañeros de viaje? ¿Por qué?

3. ¿Qué ventaja tienen el ir todos juntos?

4. ¿Creéis que uno solo hubiera podido asustar a los ladrones?

5. Por último, ¿Qué hubiera ocurrido si cada uno de ellos se hubiera encontrado con los ladrones sin la compañía de los otros animales? 
Todas las preguntas, tienen como finalidad comprobar que se ha hecho una lectura comprensiva y que todo el alumnado es capaz de respetar a sus compañeros/as y permanecer atentos a la lectura, además de saber reflexionar tras lo leído. Una vez que hayan trabajado este cuento, les propondremos que el grupo de los mayores escenifiquen la escena donde consideren que se demuestra el valor de la amistad.

\subsection{LA HONESTIDAD}

\subsubsection{Objetivos propuestos a tratar en las actividades:}

- Fomentar cualidades de atención, escucha y diálogo como medios y como clima fundamental para lograr unas relaciones interpersonales óptimas y una solución no violenta de los posibles conflictos que pueden surgir.

- Mejorar el clima de trabajo, fomentando una actitud participativa y solidaria de la comunidad educativa.

\subsubsection{Actividad 1 sobre la Honestidad:}

Para trabajar el valor de la honestidad, se utilizará la técnica de la lluvia de ideas, donde los alumnos deberán decir palabras o frases que piensen que definen el concepto de honestidad y se irán tomando notas en la pizarra. De esta manera, con la ayuda del profesorado voluntario se realizará una definición de este valor y se pegará en la pared del aula. Ahora, todos los alumnos por turnos representarán la obra teatral de Pedro y el Lobo para que los alumnos se pongan en la piel de los personajes que aparecen en esta historia, sobre todo, de Pedro, que por mentir tanto ya la gente del pueblo no le creía y concienciarles de esta manera que la mentira no conduce a nada bueno. Una vez que todos hayan participado en esta pequeña obra teatral, les daremos al grupo de los mayores unos minutos para que piensen cómo darle un giro a la historia para que se consiga el respeto de todos los ciudadanos de la aldea y qué cambio de actitud tiene que adoptar ahora Pedro para que se gane la confianza de sus vecinos, y tendrán de nuevo que escenificar la historia con sus respectivos cambios.

\subsubsection{Actividad 2 sobre la Honestidad:}

Para trabajar con los alumnos se realizarán pequeños teatrillos que representan escenas cotidianas, entre unos padres y sus hijos. En un momento de la escena, un hijo debe decidir si elige la honestidad o, por el contrario, les miente. En este momento de inflexión, los alumnos deben solventar qué acción es la más correcta. Para esto, estos teatrillos tienen un final abierto que son los propios alumnos que deben decidir cómo acabará la historia o diciendo la verdad o mintiendo. Tras los teatros, se dividirá la clase en dos y a cada grupo se le repartirá una canica. Esta canica se le da a uno de los integrantes de cada grupo, 
e irá pasando desde las manos de un niño a otro, mientras uno de cada grupo se da la vuelta y cuenta hasta 10. Una vez que este termina de contar en ambos grupos, les pregunta a tres de sus compañeros si tiene la canica. Si le pregunta al niño que tiene la canica debe ser honesto y dársela y convertirse en el que cuenta y busca la canica, y seguir la ronda. Si ninguno de los tres tiene la canica, el "buscador" repite. Si el que tiene la canica miente, pasa a ser "el buscador "durante dos rondas seguidas.

\section{EVALUACIÓN}

La evaluación que se propone para este proyecto tendrá como objetivo comprobar si este alcanza los resultados esperados, tras su puesta en marcha. Esta evaluación será llevada a cabo en tres momentos diferentes junto con una autoevaluación.

En primer lugar, se ejecutará la evaluación inicial al principio de algunas actividades mediante, por un lado, la utilización de la herramienta de trabajo grupal lluvia de ideas (brainstroming) y, por otro lado, la realización de preguntas concretas para obtener información de los conocimientos previos que poseen los alumnos sobre el valor concreto que se en ese momento se dispongan a trabajar.

En segundo lugar, para llevar a cabo la evaluación formativa de cada actividad, se utilizará una escala de observación con la que se evaluará los siguientes indicadores referidos a todos alumnos: nivel de participación, grado de cooperación entre ellos, grado de afrontación de los conflictos e interiorización final de los valores trabajados semana tras semana. Esta escala, seguirá un mismo modelo para evaluar cada semana a los alumnos.

Es preciso señalar que el indicador del nivel de participación tendrá en cuenta aspectos evaluativos como el hecho de si todos los alumnos se muestran participativos en las tareas grupales, si respetan todos los alumnos a sus compañeros de clase y si responden adecuadamente a las preguntas que se les realizan en algunas actividades. Por otro lado, en cuanto al indicador del grado de cooperación y afrontación de conflictos, se tomarán en consideración aspectos evaluativos como el hecho de si entre todos los alumnos se ha logrado crear un clima de cooperación en las actividades, si muestran iniciativa en las actividades propuestas, si los alumnos por lo general adoptan una actitud de compromiso con la actividad y, por último, se evaluará si existen conflictos entre los alumnos por ser el mejor.

Para realizar la evaluación sumativa de los alumnos, se les aplicará un cuestionario para ver si se ha conseguido el objetivo principal de este proyecto de dotar a estos alumnos de unos valores sociomorales con el fin de facilitarles una adaptación eficaz en la sociedad, mejorando, sobre todo, el clima de trabajo en la clase. De esta forma, se podrá rendir cuentas a la Cruz Roja que será la encargada de subvencionar este proyecto. A posteriori se realizará una comparación de los resultados extraídos al contrastar la herramienta de evaluación formativa (escala de observación) de los alumnos con el 
cuestionario aplicado, con el fin de comprobar si verdaderamente los alumnos han logrado interiorizar los valores presentados. Para finalizar este apartado, la persona encargada de aplicar este proyecto realizará un procedimiento de autoevaluación teniendo en cuenta los diferentes momentos evaluativos, con el fin de evidenciar si se han logrado los objetivos esperados y tomar decisiones en caso de que no se hayan conseguido, para así lograr el éxito de este proyecto.

\section{CONCLUSIONES}

Tras la puesta en marcha de este proyecto se espera que se logren los objetivos marcados que se han nombrado a lo largo de este trabajo, garantizándoles a los alumnos una buena respuesta educativa y social. Defendiendo, sobre todo, su derecho a acceder a una educación basada en unos valores básicos que les pueda ayudar a prevenir situaciones de racismo y de marginación, y compensando las carencias que tienen por vivir bajo una situación de desventaja social.

Es preciso destacar que la autoevaluación que se llevará a cabo tras su puesta en marcha ayudará a identificar cuáles son aquellos puntos débiles y fuertes de este proyecto de intervención y plantear propuestas hacia la mejora. La información que se extraiga de esta autoevaluación será útil para garantizar su éxito potencial en un futuro y seguir contribuyendo a prestar las necesidades más básicas de la atención de la población con riesgo de exclusión.

A modo de conclusión, se puede decir que las consecuencias que se pretenden con su puesta en marcha es que a diferencia de otros proyectos y planes de educación en valores llevados a cabo en la Región de Murcia, se plantee desde el organismo de la Cruz Roja que se preocupe por dar una respuesta más cercana a aquella población que lo necesite, sobre todo, a los menores garantizándoles desde este proyecto una calidad educativa que logre la inclusión e integración de todo el alumnado. Por supuesto, no dejando de lado el hecho de prestarles aquellos servicios sociales que lo precisen para la continuidad de este tipo de educación a lo más menores, e implicando en algunas acciones a la familia para que desde esta se trabajen aquellos valores socialmente aceptados y deseados. Sólo de esta manera, se propiciará un ambiente rico en valores y se prepararán a los alumnos a construir una serie de valores para adaptarse eficazmente en esta sociedad donde "todas las piezas de la estructura comunitaria, formando parte de un mismo tejido social, resistan unidas, apoyándose en las iniciativas educativas de valor, ya que esta colectividad se cohesiona cuando ese entramado se ve fortalecido" (Belmonte y Bernárdez-Gómez, 2020, p.45), favoreciendo la formación hacia la igualdad de la diferencia, a fin de ir consolidando un conocimiento real sobre la discapacidad alejado de prejuicios y falsas creencias (Mirete, Belmonte y Maquilón, 2020). 


\section{REFERENCIAS BIBLIOGRÁFICAS}

Annaut, L. (2002). Valores escolares y educación para la ciudadanía. Graó.

Ballester, F. y Calvo, A. R. (2007). Cómo elaborar planes para la mejora de la convivencia. EOS.

Bartolomé, M. et al. (2002). Identidad y ciudadanía: un reto a la educación intercultural. Narcea.

Beane, J. A. y Apple, M. (2002). Escuelas democráticas. Morata.

Belmonte, M. L. y Bernárdez, A. (2020). Respuesta social al estado de aislamiento por coronavirus, percepciones sobre educación. Revista Conhecimento Online, 3, 30-49.

Bernárdez Gómez, A.; Belmonte, M. L. (2020a). School dropout, determinants, educational policies and subsequent itineraries. Research, Society and Development, [S. 1.], 9 (10), p. e6849109234. DOI: 10.33448/rsd-v9i10.9234.

Bernárdez Gómez, A.; Belmonte, M. L. (2020b). Vulnerabilidad y exclusión educativa, una problemática postergada. Revista GETS, 3 (1), 79-94.

Bernárdez-Gómez, A. (2021). La investigación sobre trayectorias educativas. South Florida Journal of Development, 2(1), 42-53. https://doi.org/10.46932/sfjdv2n1-005

Bolívar, A. (1995). La evaluación de valores y actitudes. Anaya.

Bolívar, A. (2006). Familia y escuela: dos mundos llamados a trabajar en común. Revista de educación, $339,119-146$.

Booth,T. y Ainscow, M. (2000). Index for Inclusion: Developing Learning and Participation in Schools. Centre for Studies on Inclusive Education (CSIE).

Camps, V. (1998). La enseñanza de los valores: qué, cómo y quién. Infancia y aprendizaje, 21(82), 103 107.

Camps, V. y Giner, S. (2004). Manual de civismo. Ariel.

Cruz Roja Española. (2014). Cruz Roja. España. http://www.cruzroja.es

Esteban, M. (2003). Educación en valores. Programa para su desarrollo en la Educación Secundaria. Tendencias pedagógicas, 8, 99-108.

Fabelo, J. R. (2003). Los valores y sus desafíos actuales. Editorial José Martí.

González, A. M. (1991). El Enfoque centrado en la persona. Editorial Trillas.

González, O. (2014). Familia y escuela, escuela y familia. Guía para que padres y docentes nos entendamos. Desclée.

Hernández, P., Álvarez, M. y Aranda, A. (2017). El problema de deserción escolar en la producción científica educativa. Revista Internacional de Ciencias Sociales y Humanidades, 1, 89-112. 
Hernández Prados, M. Ángeles., y Álvarez Muñoz, J. S. . (2019). Family leisure and academic achievement. Perception of the families. ITALIAN JOURNAL OF EDUCATIONAL RESEARCH, (23), 86105. Recuperado de https://ojs.pensamultimedia.it/index.php/sird/article/view/3685

Hessen, J. y Romero, F. (1970). Teoría del conocimiento. Espasa-Calpe.

Ley Orgánica 1/1990, de 3 de octubre, de Ordenación General del Sistema Educativo (BOE nº 238, de 4 de octubre de 1990). http://www.boe.es/boe/dias/1990/10/04/pdfs/A28927-28942.pdf

Ley Orgánica 2/2006, de 3 de mayo, de Educación (BOE n 106, 3 de Mayo de 2006). https://www.boe.es/boe/dias/2006/05/04/pdfs/A17158-17207.pdf

Ley Orgánica 8/2013, de 9 de diciembre, para la Mejora de la Calidad Educativa. (BOE n 295, de 9 de Diciembre de 2013). http://www.boe.es/boe/dias/2013/12/10/pdfs/BOE-A-2013-12886.pdf

Martínez, J. B. (2006). Educación y ciudadanía. Eikasia: revista de filosofía, (6), 7-19.

Martínez, S. (2014). En busca de relaciones: encuentros compartidos. Cuadernos de Pedagogía, 444, 5052.

Mínguez, R. (2014). Ética de la vida familiar y transmisión de valores morales. Revista de Educación, $363,210-229$.

Mirete, A. B., Belmonte, M. L., y Maquilón, J. J. (2020). Diseño, aplicación y validación de un instrumento para Valorar las Actitudes hacia la Diversidad del Alumnado (VADA). Revista Electrónica Interuniversitaria de Formación del Profesorado, 23(2), 185-207.

Naval, C. y Laspalas, J. (eds.) (2000). La educación cívica hoy. Una perspectiva interdisciplinar. Eunsa. Ortega, P., Mínguez, R. y Gil, R. (1996). Valores y educación. Ariel.

Parra, J. M. (2003). La educación en valores y su práctica en el aula. Tendencias pedagógicas, (8), 69-88.

Porto, M. S. G., Almeida, A. y Jodelet, D. (2009). Re-pensando crenças e valores: sociologia representações sociais. Interdisciplinaridade e diversidade de paradigmas. Thesaurus.

Rivas, E. (1996). La educación ante la crisis de valores de la sociedad actual. Laurus, (3), 10-19.

Rodríguez, M. (2002). Respuestas educativas al desarrollo de la identidad europea. En M. Bartolomé et al., Identidad y ciudadanía: un reto a la educación intercultural (pp. 51-78). Narcea.

Santos, M. A. y Lorenzo, M. M. (2009). La participación de las familias inmigrantes en la escuela. Un estudio centrado en la procedencia. Revista de Educación, 350, 277-300.

Tierno, B. (2000). Todo lo que necesitas saber para educar a tus hijos. Plaza y Janés.

Trillo, F; Parada, A.; Bernárdez-Gómez, A. (2020). El impacto formativo de la enseñanza en la universidad: una experiencia de autoevaluación docente. Brazilian Journal of Development, 6(9). https://doi.org/10.34117/bjdv6n9-279 